Research Article

Fedor Viktorovich Vereshchagin*

\title{
Processing and visualisation of a series of monochromatic images of regions of the Sun
}

https://doi.org/10.1515/astro-2022-0004

Received Oct 31, 2021; accepted Dec 26, 2021

Abstract: In order to process and visualise the monochromatic images of regions of the Sun obtained with the CCD multichannel spectroheliograph, the freely distributed programs ImageJ and ParaView were chosen and then fine-tuned. This reduced the time required to develop the data processing and visualization software for this instrument and also made it easier to make further corrections and additions to it.

Keywords: visualisation, ImageJ, ParaView, filtergram, spectroheliograph

\section{Introduction}

The multichannel CCD spectroheliograph (Nikulin and Vereshchagin 2019) is used in the Department of Solar Physics at the ATB-1 solar tower telescope to study nonstationary phenomena in the chromosphere of the Sun. During the operation of this instrument an image of a region of the Sun is transmitted to the entrance slit of the spectrograph through the scanning prism to produce series of spectral profiles. It was necessary to develop a software in order to study the acquired three-dimensional $(\mathrm{x}, \mathrm{y}, \lambda)$ data sets.

\section{Processing and visualization of acquired data sets}

To process and visualize the obtained data, freely distributed programs were chosen: the program for image analysis and processing ImageJ (Schneider et al. 2012) and the application for data analysis and interactive visualization ParaView (Ayachit 2015).

The built-in image series processing operations, the ability to configure a user-defined menu (by adding the free ActionBar plug-in (Mutterer 2017)), and the built-in macro programming language in ImageJ made it possible to create the program to represent the acquired data sets as the series of combined images consisting of both the monochromatic image itself and the averaged spectral profile with a

Corresponding Author: Fedor Viktorovich Vereshchagin: Sternberg Astronomical Institute (SAI), Moscow State University, Moscow, 119234 Russia; Email: zverted@gmail.com wavelength mark corresponding to the image (Nikulin and Vereshchagin 2019).

The visualisation functions built into ParaView made it possible to present an acquired data sets as a tomographic image in space, where the $\mathrm{z}$ coordinate corresponded to wavelengths and points outside a certain range of values were hidden with the ability to visually adjust image display parameters.

\section{Conclusion}

The ability to use Image as a image processing library, the ability to visually adjust image display parameters in ParaView not only made it possible to process and visualize the acquired three-dimensional $(\mathrm{x}, \mathrm{y}, \lambda)$ data sets, but also reduced the time required to develop the data processing and visualization software for multichannel CCD spectroheliograph and also made it easier to make further corrections and additions to it.

Funding information: The author states no funding involved.

Conflict of interest: The author states no conflict of interest.

\section{References}

Ayachit U. The paraview guide: a parallel visualization application. Clifton Park, USA: Kitware; 2015. 
Mutterer J. 2017. Custom toolbars and mini applications with Action Bar. Figshare, https://doi.org/10.6084/m9.figshare.3397603. Nikulin IF, Vereshchagin FV. 2019. A Multichannel CCD Spectroheliograph for Studying Nonstationary Phenomena in the Chromosphere of the Sun. Instrum Exp Tech. 62(4):558-561.
Schneider CA, Rasband WS, Eliceiri KW. 2012. NIH Image to Image): 25 years of image analysis. Nat Methods. 9(7):671-675. 